\title{
Non-parametric estimation of age-related centiles over wide age ranges
}

\author{
HUI QI PAN \\ Department of Mathematics and Biostatics, Shanghai Second Medical University, Shanghai, People's \\ Republic of China \\ HARVEY GOLDSTEIN \\ Department of Mathematics, Statistics and Computing, Institute of Education, London University
}

QI YANG

Department of Mathematics and Biostatistics, Shanghai Second Medical University, Shanghai, People's Republic of China

Received October 1989; revised December 201989

\begin{abstract}
Summary. A new method for estimating age-related centile curves has been developed, which is suitable for measurement covering a wide age range. The method was used to calculate weight centile curves of 8995 children from birth to 6 years obtained by the Collaborating Centre for Physical Growth and Psychosocial Development of Children in Shanghai, China.
\end{abstract}

\section{Introduction}

Healy, Rasbash and Yang (1988) presented a method for distribution-free estimation of a set of age-related centiles. The method is satisfactory for weight, circumferences, etc., whose distributions at a fixed age are often non-Gaussian. In practice, when the age span is wide, for example for childrens' stature from birth to 6 years old, or when the velocity of growth changes markedly over the age range, this method tends not to be satisfactory. This has led us to propose a new method by extending the procedure of Healy et al.

\section{Procedure}

The method of Healy et al. (1988) involves two steps. The first step is to obtain 'raw' centile estimates as follows. We define an age 'window', typically containing at least 50 data points. Starting from one end of the age scale this window is moved along the scale, one point at a time, to give overlapping sets of points. For each such set a ranking method estimates the centiles, giving an initial set of 'raw' centiles. Using these initial estimates, a polynomial of sufficiently high order is fitted to each centile. The intercept, linear, quadratic, etc., polynomial coefficients are then separately 'smoothed', effectively by regressing them on the centile normal equivalent deviates (NED). The final smoothed centiles are predicted from these values.

\section{Smoothing the initial estimates}

The raw estimates are as obtained by Healy et al. (1988) for the whole age range. These estimates are irregular and need to be smoothed to provide centile curves.

It is assumed that the curves can be fitted by a polynomial of degree $p$. If $t$ denotes age and $Y_{i, t}$ the smoothed value of the $i$ th centile, we have

$$
Y_{i, t}=a_{0, i}+a_{1, i} t+\ldots+a_{p, i} t^{p}+a_{p+1, i}\left(t-c_{1}\right)_{+}^{p}+\ldots+a_{p+m-1, i}\left(t-c_{m-1}\right)_{+}^{p}
$$


where

$$
\begin{aligned}
\left(t-c_{l}\right)_{+}^{p} & =\left(t-c_{l}\right)^{p} & \text { when } & t>c_{l} \\
& =0 & \text { when } & t \leq c_{l} \quad l=1,2, \ldots, m-1
\end{aligned}
$$

and $c_{l}$ is the $l$ th cut or join point between the age groups with $c_{1}<c_{2} \ldots<c_{m-1}$. Of course, when $m=1$, the new method is the same as that presented by Healy et al. For example, with one join point and polynomials of degree 3 we obtain

$$
\begin{array}{cr}
Y_{i, t}=a_{0, i}+a_{1, i} t+a_{2, i} t^{2}+a_{3, i} t^{3} & \text { when } t \leq c_{1} \\
=a_{0, i}+a_{1, i} t+a_{2, i} t^{2}+a_{3, i} t^{3}+a_{4, i}\left(t-c_{1}\right)^{3} & \text { when } t>c_{1}
\end{array}
$$

It is clear that the two components of the curve join smoothly at the join point, and this is generally true for curves of the form (1). The value of $m$ and $c_{l}$ can be chosen after inspection of the data and using existing knowledge (see Discussion).

The coefficients in equation (1) are now regressed on a polynomial function of the NEDs $Z_{i}$ of degree $q$.

$$
a_{j, i}=b_{j, 0}+b_{j, 1} Z_{i}+\ldots+b_{j, q_{j}} Z_{i}^{q_{j}}+e_{j, i} \quad j=0,1, \ldots p+m-1
$$

Combining (1) and (2) leads to a composite linear model for the initial centile curves whose coefficients can be estimated using ordinary least-squares (OLS) analysis. The values of $p$ and $q_{l}$ still have to be determined and in the next section we give some examples. In general, the value of $q_{l}$ will usually be higher for the low-order coefficients and may be zero for high-order ones. Some exploration will generally be needed to obtain optimum values for $p$ and $q_{l}$. A test of fit can be obtained by comparing the percentages of data points that fall between the centile curves for sub-ranges, with their expected values.

\section{Examples}

\section{Data}

In general, more than three centiles, typically the 3rd, 10th, 25th, 50th, 75th, 90th and 97 th, would be estimated. Here, for the purpose of illustration, only the 3rd, 50th and 97 th weight centile curves were calculated for 4690 male and 4305 female children from birth to 6 years old. These children were random samples from Shanghai and five other provinces in the southeast of China, from rural and urban districts. The data were obtained by the WHO Collaborating Centre for Physical Growth and Psychosocial Development of Children in Shanghai, China, the staff having been specially trained to ensure validity under the guidance of WHO, Maternal and Child Health Division, Geneva.

\section{Results}

Many combinations of $p, q, m$ and $c$ have been tried and four examples for discussion are presented.

Example 1: $p=3, m=2, c_{1}=12$ months, $q=2,2,1,0,0$, that is, $q_{0}=2, q_{1}=2, q_{2}=1$, 


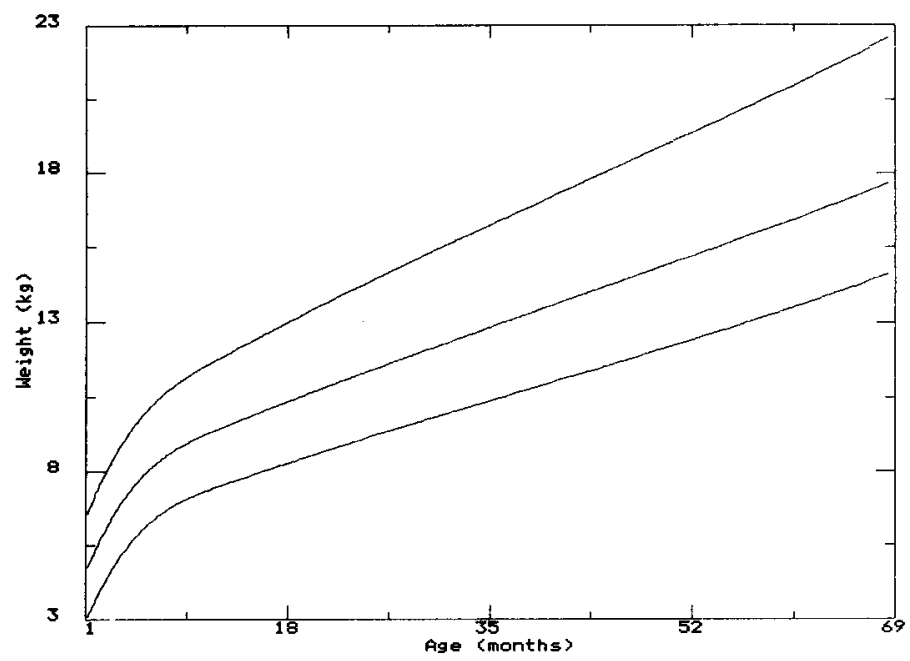

Figure 1. Example 1: 3rd, 50th and 97th centiles for males with $p=3, m=2, c_{1}=12, q=2,2,1,0,0$.

Table 1. Example 1: percentage for each subrange of age.

\begin{tabular}{|c|c|c|c|c|c|c|}
\hline & \multicolumn{6}{|c|}{ Age (months) } \\
\hline & $\geq 1 \cdot 5$ & $\geq 12$ & $\geq 24$ & $\geq 36$ & $\geq 48$ & $60-67$ \\
\hline$<\mathrm{p} 3$ & $2 \cdot 70$ & $3 \cdot 78$ & $3 \cdot 23$ & $1 \cdot 69$ & $2 \cdot 22$ & $3 \cdot 61$ \\
\hline p3-p97 & $94 \cdot 49$ & $92 \cdot 86$ & $92 \cdot 90$ & $95 \cdot 78$ & $95 \cdot 15$ & $92 \cdot 78$ \\
\hline$>$ p 97 & $2 \cdot 82$ & $3 \cdot 37$ & $3 \cdot 87$ & $2 \cdot 53$ & $2 \cdot 63$ & $3 \cdot 61$ \\
\hline Total & $100 \cdot 0$ & $100 \cdot 0$ & $100 \cdot 0$ & $100 \cdot 0$ & $100 \cdot 0$ & $100 \cdot 0$ \\
\hline$N$ & 1669 & 980 & 465 & 474 & 495 & 277 \\
\hline
\end{tabular}

$q_{3}=0, q_{4}=0$, for 4690 male children, and there is one join point at 12 months. The full model is

$$
Y_{i, t}=\left(b_{00}+\mathrm{b}_{01} Z_{i}+b_{02} Z_{i}^{2}\right)+\left(b_{10}+b_{11} Z_{i}+b_{12} Z_{i}^{2}\right) t+\left(b_{20}+b_{21} Z_{i}\right) t^{2}+b_{30} t^{3}+b_{40}(t-12)_{+}^{3}
$$

The smoothed curves are shown in figure 1 . The number of data points that fall between the centile curves are given in table 1 .

Example 2: $p=3, m=2, c_{1}=12$ months, $q=2,1,1,0,0$, for 4305 female children. The model is

$$
Y_{i, t}=\left(b_{00}+b_{01} Z_{i}+b_{02} Z_{i}^{2}\right)+\left(b_{10}+b_{11} Z_{i}\right) t+\left(b_{20}+b_{21} Z_{i}\right) t^{2}+b_{30} t^{3}+b_{40}(t-12)_{+}^{3}
$$

The smoothed curves with initial centile estimates are shown in figure 2 . The counts in each category are given in table 2 . 


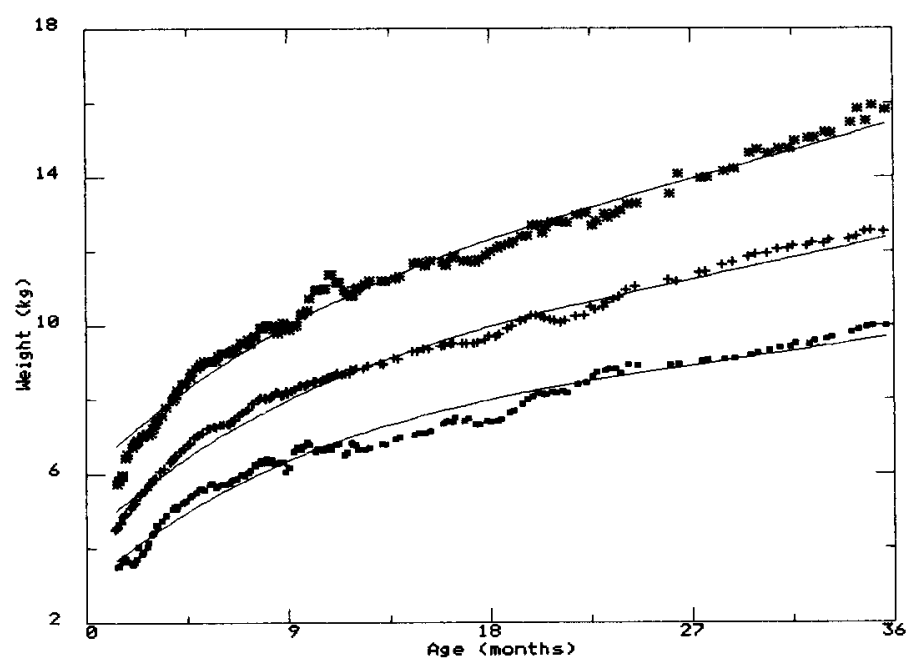

Figure 2. Examples 2: 3rd, 50th and 97th centiles for females with $p=3, m=2, c_{1}=12, q=2,1,1,0,0$.

Table 2. Example 2: percentage for each subrange of age.

\begin{tabular}{ccccccc}
\hline & \multicolumn{7}{c}{ Age (months) } \\
\cline { 2 - 7 } & $\geq 1 \cdot 5$ & $\geq 12$ & $\geq 24$ & $\geq 36$ & $\geq 48$ & $60-67$ \\
\hline$<\mathrm{p} 3$ & $2 \cdot 59$ & $3 \cdot 57$ & $2 \cdot 17$ & $3 \cdot 32$ & $1 \cdot 84$ & $2 \cdot 55$ \\
$\mathrm{p} 3-\mathrm{p} 97$ & $94 \cdot 43$ & $93 \cdot 43$ & $95 \cdot 22$ & $94 \cdot 08$ & $95 \cdot 16$ & $94 \cdot 91$ \\
$>$ p97 & $2 \cdot 98$ & $3 \cdot 00$ & $2 \cdot 61$ & $2 \cdot 61$ & $3 \cdot 00$ & $2 \cdot 55$ \\
Total & $100 \cdot 0$ & $100 \cdot 0$ & $100 \cdot 0$ & $100 \cdot 0$ & $100 \cdot 0$ & $100 \cdot 0$ \\
$N$ & 1543 & 868 & 460 & 422 & 434 & 275 \\
\hline
\end{tabular}

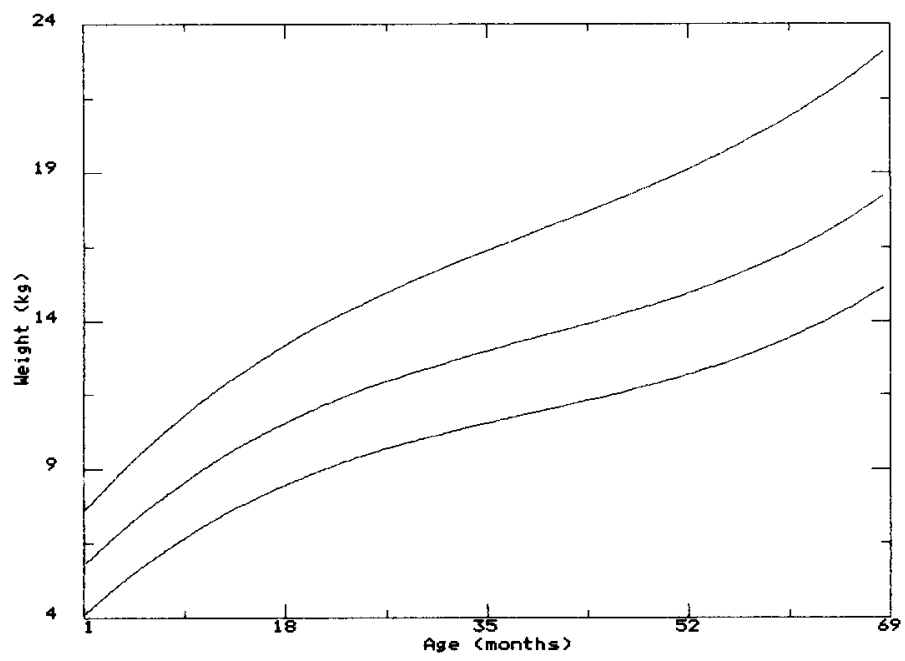

Figure 3. Example 3: 3rd, 50th and 97th centiles for males with $p=3, m=1, q=2,2,1,0$. 
Table 3. Example 3: percentage for each subrange of age.

\begin{tabular}{rcccccc}
\hline & \multicolumn{7}{c}{ Age (months) } \\
\cline { 2 - 7 } & $\geq 1 \cdot 5$ & $\geq 12$ & $\geq 24$ & $\geq 36$ & $\geq 48$ & $60-67$ \\
\hline$<$ p3 & $2 \cdot 22$ & $5 \cdot 10$ & $4 \cdot 95$ & $1 \cdot 48$ & $1 \cdot 62$ & $4 \cdot 69$ \\
p3-p97 & $93 \cdot 59$ & $91 \cdot 84$ & $91 \cdot 83$ & $95 \cdot 99$ & $95 \cdot 56$ & $92 \cdot 06$ \\
$>$ p97 & $4 \cdot 19$ & $3 \cdot 06$ & $3 \cdot 23$ & $2 \cdot 53$ & $2 \cdot 83$ & $3 \cdot 25$ \\
Total & $100 \cdot 0$ & $100 \cdot 0$ & $100 \cdot 0$ & $100 \cdot 0$ & $100 \cdot 0$ & $100 \cdot 0$ \\
$N$ & 1669 & 980 & 465 & 474 & 495 & 277 \\
\hline
\end{tabular}

Example 3: $p=3, m=1, q=2,2,1,0$, for 4690 male children. Here, $m=1$ means there is no join point. The model is

$$
\left.Y_{i, t}=\left(b_{00}+b_{01} Z_{i}+b_{02} Z_{i}^{2}\right)+b_{10}+b_{11} Z_{i}+b_{12} Z_{i}^{2}\right) t+\left(b_{20}+b_{21} Z_{i}\right) t^{2}+b_{30} t^{3}
$$

The smoothed curves are shown in figure 3 and the counts are given in table 3.

Example 4: $p=4, m=1, q=2,1,1,0,0$, for 4305 female children. The model is

$$
Y_{i, t}=\left(b_{00}+b_{01} Z_{i}+b_{02} Z_{i}^{2}\right)+\left(b_{10}+b_{11} Z_{i}\right) t+\left(b_{20}+b_{21} Z_{i}\right) t^{2}+b_{30} t^{3}+b_{40} t^{t}
$$

The curves with initial centile estimates are given in figure 4 and the counts are in table 4.

The curves for males and females with one join point appear to fit the data well. This is clear from figures 1 and 2 and from tables 1 and 2 where the percentages in the centile bands do not deviate appreciably from their expectations and a formal test of fit yields a non-significant result at the $5 \%$ level. By contrast, in model 3 with no join point, figure 3 shows an upward curvature at the oldest ages and an overall poor fit as is seen in table 3. Example 4 also fits a single curve, but with a fourth-order term in order to introduce an extra parameters, thereby giving the same number of parameters as models 1 and 2 . It provides a better fit than model 3 at the older ages but a poor fit at the younger ones, which is also apparent from table 4.

\section{Discussion}

We have demonstrated how one of the limitations of the Healy et al. (1988) method for centile estimation can be overcome by introducing extra parameters into the polynomial smoothing. However, the approach of simply increasing the order of the polynomial does not work well in general as is illustrated in our examples. The proposed method allows a wide age range to be fitted using relatively low-order polynomials. The choice of the number and placing of join points in this method is a matter for experimentation. In our examples several ages were tried for a single join point in the range 9-12 months. This range was chosen because it is where the growth velocity decreased rapidly. The results were not sensitive to the precise choice of age.

A generalization of the procedure described here (Goldstein and Pan 1990) allows different order polynomials to be fitted within each age range and also allows simultaneous estimation for different population subgroups. Both procedures can be fitted using the GROSTAT II program (Rasbash 1989). 


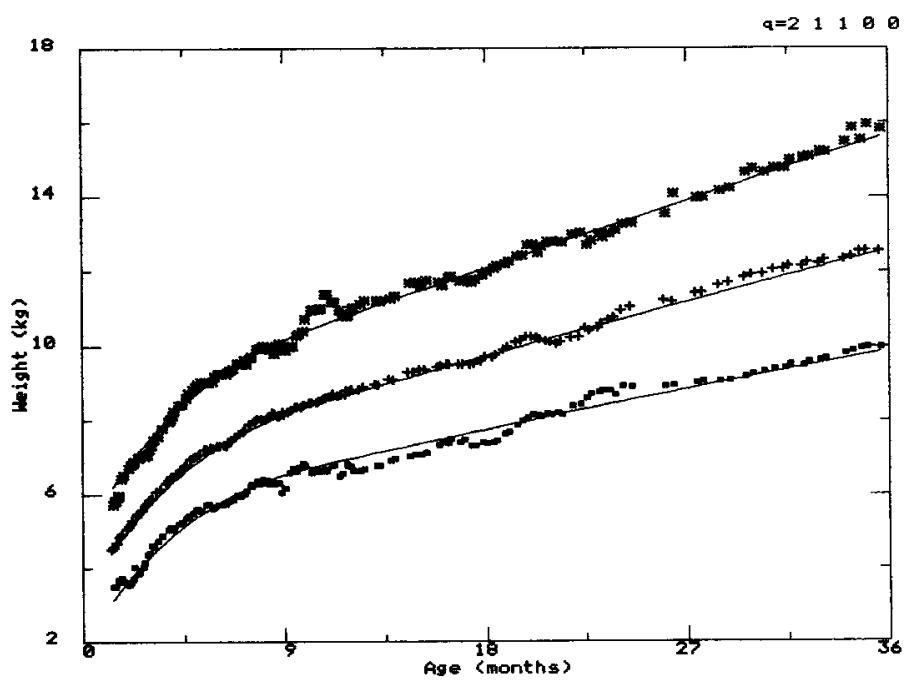

Figure 4. Example 4: 3rd, 50th and 97th centiles for females with $p=4, m=1, q=2,1,1,0,0$.

Table 4. Example 4: percentage for each subrange of age.

\begin{tabular}{|c|c|c|c|c|c|c|}
\hline & \multicolumn{6}{|c|}{ Age (months) } \\
\hline & $\geq 1 \cdot 5$ & $\geq 12$ & $\geq 24$ & $\geq 36$ & $\geq 48$ & $60-67$ \\
\hline$<\mathrm{p} 3$ & $2 \cdot 20$ & $5 \cdot 07$ & $1 \cdot 96$ & $2 \cdot 84$ & $2 \cdot 07$ & $2 \cdot 91$ \\
\hline p3-p97 & $93 \cdot 84$ & 93.09 & $94 \cdot 57$ & $94 \cdot 31$ & $95 \cdot 16$ & $94 \cdot 91$ \\
\hline$>$ p97 & 3.95 & $1 \cdot 84$ & $3 \cdot 48$ & $2 \cdot 84$ & $2 \cdot 76$ & $2 \cdot 18$ \\
\hline Total & $100 \cdot 0$ & $100 \cdot 0$ & $100 \cdot 0$ & $100 \cdot 0$ & $100 \cdot 0$ & $100 \cdot 0$ \\
\hline$N$ & 1543 & 868 & 460 & 422 & 434 & 275 \\
\hline
\end{tabular}

\section{Acknowledgements}

We are most grateful to $\mathrm{Dr}$ Guo $\mathrm{Di}$, and Professor Michael Healy who have provided invaluable comments on the paper. Many thanks are due to Mr Jon Rasbash and Mr Robert Prosser who assisted in this effort. We are also grateful to the staff of the WHO Collaborating Centre in Shanghai for the data. This work was partly carried out while the first author was in receipt of a WHO fellowship at the Institute of Education, University of London.

\section{References}

Healy, M. .J. R., RASBash, J. and YANG, M., 1988, Distribution-free estimation of age-related centiles. Annals of Human Biology, 15, 17-22.

Department of Probability and Statistics at the Chinese Science Iinstitute, 1979, Probability, Statistics and Computation. (Beijing: Science Press), 175-181.

Goldstein, H. and PAN, H., 1990, Percentile smoothing using piecewise polynomials, with covariates. (Submitted for publication).

Rasbash, J., 1989, GROSTAT II: A Program for the Construction of Age Related Centiles. WHO Collaborating Centre on Growth and Development, University of London, London WC1, England.

Address for correspondence: Hui Qi Pan, Department of Mathematics Statistics and Computing, Institute of Education, University of London, 20 Bedford Way, London WC1H OAL. 
Zusammenfassung. Es wurde eine neue Methode zur Schătzung der altersverknüpften Perzentilkurven entwickelt, die für Maße geeignet sind, die eine breite Altersspanne abdecken. Sie wird benutzt für die Berechnung von Gewichtsperzentilkurven von 8.995 Kindern von der Geburt bis 6 Jahre aus dem Kooperationszentrum für Körperwachstum und psychosoziale Entwicklung von Kindern in Shanghai in China.

Résumé. Une nouvelle méthode d'estimation des courbes de centile en fonction de l'âge a été élaborée, afin de convenir à des mensurations échelonnées sur une longue gamme d'âge. Elle est utilisée pour le calcul des courbes de centile du poids de 8995 enfants, depuis leur naissance jusqu'a 6 ans, qui ont été rassemblées par le Centre de Collaboration pour la Croissance Physique et le Développment Psychosocial de Shangai (Chine). 